\title{
Ethical issues in assistive ambient living technologies for ageing well
}

\author{
Francesco Panico ${ }^{1}$ (D) - Gennaro Cordasco ${ }^{1,2} \cdot$ Carl Vogel $^{3} \cdot$ Luigi Trojano $^{1}$. \\ Anna Esposito ${ }^{1,2}$
}

Received: 21 December 2019 / Revised: 29 June 2020 / Accepted: 9 July 2020 /

Published online: 17 July 2020

(C) The Author(s) 2020

\begin{abstract}
Assistive Ambient Living (AAL) in ageing refers to any device used to support ageing related psychological and physical changes aimed at improving seniors' quality of life and reducing caregivers' burdens. The diffusion of these devices opens the ethical issues related to their use in the human personal space. This is particularly relevant when AAL technologies are devoted to the ageing population that exhibits special bio-psycho-social aspects and needs. In spite of this, relatively little research has focused on ethical issues that emerge from AAL technologies. The present article addresses ethical issues emerging when AAL technologies are implemented for assisting the elderly population and is aimed at raising awareness of these aspects among healthcare providers. The overall conclusion encourages a person-oriented approach when designing healthcare facilities. This process must be fulfilled in compliance with the general principles of ethics and individual nature of the person devoted to. This perspective will develop new research paradigms, paving the way for fulfilling essential ethical principles in the development of future generations of personalized AAL devices to support ageing people living independently at their home.
\end{abstract}

Keywords Assistive ambient living $\cdot$ Elderly $\cdot$ Ethics $\cdot$ Ageing $\cdot$ Healthcare

\section{Introduction}

Assistive Ambient Living (AAL) technology encompasses any device, software, or humanmachine equipment helping individuals in everyday activities (including work) in spite of

Francesco Panico

francesco.panico@unicampania.it

1 Department of Psychology, University of Campania "Luigi Vanvitelli", Caserta, Italy

2 International Institute for Advanced Scientific Studies (IIASS), Vietri sul Mare, Italy

3 Trinity College Dublin, Dublin, Ireland 
environmental challenges or personal physical and cognitive impairments. AAL involves the application of organized knowledge and skills, procedures and systems whose main purpose is to preserve or increase an individual's functioning and autonomy, and ultimately to promote well-being and improve quality of life [25]. Instances of AAL applications are: i) mobility aids to help people moving in their environments [13], ii) cognitive aids, to assist people with memory and attention impairments [50], iii) voice recognition software, screen readers, and screen magnifier applications, to help people with limited mobility and sensory impairments in the use of computers and mobile devices [31, 52], and iv) utensils to help perform daily-life tasks such as cooking, dressing, and grooming [17, 42, 46].

AAL has been applied for assisting ageing related psychological and physical changes in order to reduce the burden of elders' caregivers [36] and improve seniors' quality of life by providing physical and cognitive support in daily tasks. Indeed, older people generally wish to continue living in their own homes rather than be relocated into residential institutions. However, assisting them at home often implies considerable burdens in terms of medical and social care costs associated with the assistance of such people.

In this framework, AAL applications might help to preserve older people's autonomy in their own homes and simultaneously to develop caregivers' feeling of being supported in providing them care; this will ultimately produce an overall growth of well-being [15]. AAL applications for helping ageing individuals in their homes include health-related technologies, ranging from physiological monitoring (i.e. collection and analysis of physiological parameters), to social interaction monitoring (i.e. collection and analysis of data related to social exchanges such as phone calls or visitors), and cognitive assistance (i.e. technologies encompassing automated or self-initiated reminders and other cognitive facilitations such as medication reminders, management tools, and instructions about how to use an appliance; for a review of all the mentioned applications see Demiris et al. [16]). In order to reduce costs for health services, engage people in the management of their own self-care, and sustain alternatives to under-pressure health care systems, the European Commission (EC) and several governmental agencies have funded a number of projects aimed at developing assistive devices and robots devoted to seniors' assistance and at assessing feasibility of implementation of such devices in living environments. In this context, it is worth it to mention the ULISSE, ENRICHME, and EMPATHIC projects (listed in the time order they were funded), in whose formulation the attention toward ethical aspects is particularly relevant. Firstly, in Italy, the Ministry of Health co-financed the ULISSE project (2003-2006; http://old.iss.it/esps/ index.php?lang=1\&id=139\&tipo=8), a multicenter study aimed at improving knowledge of elders' needs (particularly of elders cared by healthcare services) and the quality of care provided by health care services [32]. The ULISSE project highlighted the inappropriateness of these services and the demand for more integrated services for cure provisions, management of health conditions and of mood and communicative disorders [32]. To this aim, ULISSE identified flaws in the provided care and underlined the need of more appropriate and effective care releases. The EC-funded ENRICHME (2015-2018; https://cordis.europa.eu/project/id/ 643691) project attempted to develop an interactive robot able to move autonomously in an elderly person's house and monitor the activities and some physiological parameters of mild cognitive impaired (MCI) seniors, "discreetly" [49], i.e. avoiding invasive conduct and introducing concerns about privacy and ethical issues. More recently, the EC-funded EMPATHIC project (2017-2020; https://cordis.europa.eu/project/id/769872) aims to develop virtual assistive and empathic coaches to assist elderly people living independently at their homes, employing remote non-intrusive technologies to extract physiological markers of 
emotional states in real-time for online adaptive responses to the needs of the coached people [55]. In EMPHATIC an entire work-package was devoted to ethical issues in order to ensure a responsible development of the proposed technology under the Responsible Research and Innovation (RRI) umbrella. The concept of RRI was formulated in the Rome Declaration of the European Council (2014) but attained an imperative status only during the implementation of the EC Horizon 2020 research programme where it was emphasized that all Research and Innovation activities must be of aligned "to the values, needs and expectations of society (...)" [and] "must consider (...) the respect of human dignity, freedom, democracy, equality, the rule of law and the respect of human rights, including the rights of persons belonging to minorities" (The Rome Declaration of the European Research Council 2014).

Indeed, hidden in the promise of improving elders' quality of life there are several ethical issues to be addressed, associated with the psychological and practical implications of spreading these technologies into elders' individual personal space, and this is the awareness this paper seeks to raise. In spite of the importance of these aspects in the implementation and success of AAL interventions, only a handful of studies addressed this specific topic. Indeed, a literature search (as of May the 1st, 2020) with the key words Assistive Ambient Living $x$ Ethics $x$ Elderly retrieved only one full-length paper from PubMed [51] and, from Scopus, one book chapter [11], one conference paper [12] and one conference review [1].

The present paper aims to discuss the psychological aspects connected to the introduction of AAL in the life of the elderly, the obstacles that such introduction usually meets when being integrated in the life of an elder, and the ethical issues that follow.

\section{The elderly}

Ageing can be described as the time-related physiological process responsible for the decline of physiological functions crucial for survival and fertility (biological ageing) [26]. However, the ageing process is also associated to the deterioration of psychosocial functionalities. In this perspective, ageing develops as a complex and individualized process that involves decline at biological, psychological, and social levels [20]. Indeed, ageing-related biological changes are likely to affect mood, attitude to the environment, physical conditions, and social activities, and calling for the need to develop social interventions for these facets of ageing. Psychological ageing refers to the individual's subjective perception, appraisal and reaction to cognitive and intellectual changes due to the biological ageing process. Social ageing reflects the limitations of the role an old person occupies in the society, how she/he perceives this process and how society in turn sees it [20].

In the last decades, the increase in life expectancy [3, 54] has led to a parallel expansion of the way biological, psychological and social aspects associated with ageing are experienced.

The Life-Span Theory developed by Baltes $[4,5]$ has shed light on both gains and losses occurring during ageing and has reconsidered the learning process functioning in the life span. According to this theory $[4,5]$ the individual's cognitive, psychological and emotional characteristics, develop over the whole course of life, including the elderly. Growing and ageing are characterized by multiple physical and cognitive changes. Some of these are continuous, as the build-up of autobiographical experiences and acquisition of new knowledge. Others are discontinuous and novel in the sense that they have not been previously experienced, as age-related losses of functional competences due to motor and cognitive decline. Each stage of the life cycle introduces new challenges and new physical and cognitive 
settings requiring individuals to adapt to new biological, psychological and social conditions. To counteract physical, psychological, and social changes associated with ageing, some of which involve a diminishing of formerly robust capacity, elders exploit redeeming strategies to compensate for the detriment of some physical, psychological, and social abilities, to select areas of interests where to invest, and to optimize their expertise in some restricted fields [4, 5]. More thoroughly, compensation refers to the use of tools, strategies or technologies allowing individuals to minimize physical and cognitive shortcomings that may impede the fulfillment of an activity. Selection refers to the narrowing of personal interests only to activities considered most significant for the person. Optimization implies investing efforts in the execution of selected activities. For example, the seniors' decline in declarative memory [48] might require using notes and agendas to remember important information (compensation), as well as being engaged in limited and more familiar and tailored activities (selection) while devoting more efforts to encode actively information (optimization).

Within this framework, the age-related shortcomings represent an element of discontinuity in the individual life cycle requiring the initiation of new innovative and adaptive processes. The introduction of AAL in the seniors' life space may represent an adaptive process possibly allowing to compensate and optimize daily activities in order to ensure autonomous ageing [24] in the grounds of their personal and social spaces.

\section{Ethical issues associated to AAL in the elderly}

As AAL becomes more widespread in the ageing settings, several ethical issues associated with its use arise. The present section examines these issues and the underlying argumentation, and then the focus moves to the embodiment of these principles in AAL applications.

\subsection{Ethical principles in AAL}

Kitchner [30] described five ethical principles, exemplified in the concepts of autonomy, beneficence, non-maleficence, fidelity, and justice, to guide the implementation of assistive technology research and assistive clinical applications. Cook [14] also addressed utility and independence: utility may be seen as the requirement that AAL devices provide beneficial functionality to the user; independence may be understood in terms of the devices supporting users in functioning socially and in solitude without constant intervention from other human carers. These principles are in continuity with the medical ethics literature [6].

The principle of autonomy is related to the requirement of non-interference, to allow people to live their lives in the same way they had always done before, respecting their rights and inclinations, i.e. autonomy is the privilege and entitlement of human beings to fully determine and orient the course of their own lives. Autonomy, in the context of AAL technologies, means to ensure that the assistive devices developed for elderly care must not interfere with the will of the person they are caring for [51]. While the principle of autonomy is related to that of noninterference, they are not identical principles: AAL technologies must be useful and acceptable but without leading their users to yield their autonomy to them. It is important that people be encouraged to maintain responsibility for their decisions.

The concept of beneficence associated with AAL applications requires that any applications should be only for the benefit of the individual it is devoted to. In line with the principle of beneficence, the obligation from health-care actors only stops when there is no further AAL 
intervention to be used to support the individual's residual physical and cognitive ability [14]. The focus here is on the extent of the improvement the individual can reach, in the respect of her/his autonomy and dignity.

The principle of non-maleficence implies that AAL applications must never harm the individuals they are supporting. The difficulty here is related to the definition of harm, which must be intended not only as a physical damage. Harming can be associated to psychological costs (as confusion or emotional distress) in using AAL applications as individuals could see themselves as disabled or limited in their own will. In this context, AAL applications must be proposed only if no harm can derive from them, including distress caused by forcing patients to use them, neglecting their wills, decisions, self-esteem, integrity and preferences [27, 51]. Thus, beneficence and non-maleficence together represent fundamental concepts to any homecare intervention focusing on AAL. Yet, both principles face challenges from the fact that any intervention may have unintended consequences.

The principle of fidelity involves the concepts of faithfulness, correctness, and authenticity of reciprocal commitments. Under this principle, users must be in the position of trusting the devices and have confidence in the human-machine relationship, which in turn must take care of not threatening the user's willingness to interact with the device under whatever assumption (even the promise of improving the current physical and psychological state).

Finally, the principle of justice deals with the need to treat individuals fairly, respecting their rights, including the entitlement to take questionable decisions, as well as accounting for diversity and individual differences. This means to ensure fairness in individual, interpersonal, organizational and societal contexts.

Although the value and theory of these principles are of no difficult understanding, their fulfillment can be challenging when designing and implementing AAL technologies. It is crucial to highlight how these principles can be at odds in some circumstances and how they can be (mis)interpreted due to the multiple shades of meaning that they bring with them. For instance, the principle of beneficence would require using AAL technologies for any individual who needs assistance, but this might conflict with the privilege of a person in living independently and the choice of by whom or what she/he wants to be assisted, i.e. the principle of autonomy. Moreover, as far the principle of fidelity is concerned, covert AAL behaviors may be designed with inherent beneficence, yet to the extent that they are hidden from the users they could be at odds with the principle of fidelity. An additional conflict may also arise between the need for minimizing the possible harm [35] in a period of advancing scientific knowledge and healthcare facilities. Even more challenging can be the attempt to solve these conflicts. This requires taking at the center of designing and implementing AAL technology the concept of human dignity, as no social, caring or research interests have priority with respect to the dignity of individuals [43].

\subsection{Embedding ethics in AAL}

Concrete instances of the above-mentioned principles are exemplified by several recent EC projects in AAL. For instance, autonomy in asking for help and fidelity in terms of nonobtrusive interactions between the user and AAL technology, have inspired the development of Council of Coaches project [45; https://cordis.europa.eu/project/id/769553]. This project aimed at making the users free to choose to (actively or passively) join the suggested course of action for improving their psychological and mental health; this is achieved providing specific information to promote self-confident decision makings in health contexts, by means of 
leading edge spoken dialogue interaction technologies. Fairness in individual and intrapersonal societal exchanges (i.e. justice) were two major measures of the ARIA-VALUSPA project [56; https://cordis.europa.eu/project/id/645378] in which emphasis was given to the need of capturing both verbal and non-verbal components of dialogue and what these signals reveal about the users' mental state (cognitive, affective, social) in order to react appropriately through emotionally colored social signals. The purpose of avoiding harm and preserving beneficence of the intervention prompted the EMPATHIC project [55; https://cordis.europa.eu/project/id/ 769872], and this was achieved by extracting behavioral markers of emotional states and mood changes in real-time to allow the AAL device to promptly adapt and support its users. Far from providing a systematic analysis of each of them, these examples represent only a few of the thousand projects funded by the EC accurately reflecting the principles described above.

However, the application of these general principles when offering AAL solutions to vulnerable people is not sufficient to cover all the related ethical issues. Recent literature has suggested that people interpret the use of care robots in care settings depending on how they perceive the boundaries of the ethical landscape in aged-care [57]. Vandemeulebroucke et al. [57] highlighted that an ethical analysis on care robot employs democratic spaces, in which all the actors involved in aged-care, especially care recipients, should have a voice. The way older adults experience and perceive socially assistive robots depends on several aspects, among these 1) the role of care robots in the individual life, 2) the technical and human aspects of the interaction between older adults and care robots, and 3) robot's physical appearance [58]. A very recent theoretical framework for understanding ethical aspects in care robot's applications comes from a focus group study on older adults [59]. This study revealed that seniors' perception of ethics of care robots is structured along three lines: a) care robots as elements of a technological and societal evolution, b) robot's embeddedness in aged-care dynamics, and c) embodiments of ethical considerations, i.e. users' views about the ethics of robot applications on a relational level (e.g. necessity of being able to turn off the robot and the right to decline robot's suggestions). Understanding robots as elements of technological and societal evolution may persist at odds with understanding this evolution as democratic, in that while elders may enjoy that academic roboticists appear to care what features users require and which features are deemed intrusive, they will have had a lifetime of experience of technology manufacturers unilaterally defining what features will be available or not and how terms of service will operate, and so on, partly through application of the "planned obsolescence" model of product development.

These general considerations about care robots can be extended to different AAL applications even though some of them may not require the interaction with humans. However, for those involving interactions, ethical issues should be considered to different degrees of significance. For instance, some AAL technologies involve the use of devices with no physical presence, thus being less intrusive in the domestic space as compared to care robot applications. Nevertheless, this apparent advantage has been not yet closely investigated from an ethical point of view.

Another important area of investigations relates to the assessment of positive and negative qualities individuals can attribute to AAL applications, i.e. their possible advantages, disadvantages, and raised concerns at a personal and societal level $[11,19]$. To this aim, there is a need of:

1) Evaluating the societal impact of AAL applications, in terms of advantages and drawbacks. For example, even if AAL applications can be beneficial in improving some individual's functionalities that deteriorate through ageing, and provide support to both 
elders and caregivers, the commonly shared concern that AAL applications reduce care services, decrease human contact and cool down relationships should be assessed carefully.

2) Assessing the extent to which seniors agree to have their personal space occupied by AAL applications [11,57-59]. The concern here is related to the possibility that AAL may be installed in private personal environments without obtaining the consent, or even without awareness of the involved individual. This is particularly relevant when AAL applications are serving individuals with moderate-to-severe cognitive impairments, such as old people with dementia [9].

3) Respecting privacy. AAL devices devoted to the monitoring of physiological and psychological functionalities require the recordings and storing of personal data, for remote caregiving. Some AAL devices raise privacy issues for those who visit or are visited by the device host, as well, whether in a professional, personal or casual capacity. Issues of how to ensure that such information remains confidential for doctors and caregivers and is not misused or lost must be appraised. To this aim, Bedaf et al. [7] recently showed that, which data are stored and who has access to these data, represent a common concern for elderly individuals, caregivers and healthcare professionals.

4) Restraining over-usage. The possibility of over-using AAL applications with the risk to produce a loss of personal skills should be avoided and individuals should be encouraged to focus on their abilities rather than to depend exclusively on their (humans or robotic) helpers. This concern seems to characterize mainly caregivers and healthcare professionals promoting the fulfillment of conjoint tasks involving seniors and devices, in order to keep them active, rather than seniors who might prefer a rather passive and obedient device [7].

A further line of interest focuses on some idiosyncratic aspects of the individual and the environment. It is indeed important to consider users' motivations, perspectives, and preferences in introducing AAL applications in their daily life, as these characteristics may change from person to person and from context to context $[11,53]$. Some qualitative and quantitative aspects of the disability the AAL applications are called to support must be taken into account. As an instance, assisting an individual under stable conditions, e.g. a person with acquired motor deficits and no concomitant cognitive decline, is different from assisting a person with neurodegenerative disorders, i.e. with fluctuating physical and psychological impediments in daily routine. The involvement or the possibility to involve other carers can help when an AAL device is going to be introduced in a person's daily routine. An evaluation of the individual living space and social context, her/his idiosyncrasies, and an accurate assessment of the available alternatives must be at the core of building an intervention tailored on the individual's needs, shortcomings and wills, in order to prevent the failure of the intervention itself.

The above described theoretical principles $[14,30]$ raise a further major issue about ethics of AAL applications, in relation to the risk that the helping purpose inherent in these devices could result in over-help, i.e. a form of intrusion in the users' autonomy with a consequent negative evaluation and rejection of the device [47]. The ability to monitor the social signals regulating on-going supporting processes in real-life interactions [18, 33] should be an essential feature of the next generation of AAL devices for preventing the risk of over-help and psychological distance and promoting tailored help and real acceptance. 


\section{Conclusions and future directions}

AAL represents a valuable opportunity to improve quality of life of people with limited autonomy and of their caregivers. However, the introduction of AAL devices in the private individual space raises psychological consequences and ethical issues, possibly interfering with the success of the intervention. The main conclusions and future directions are described in the following paragraphs.

\subsection{Tailoring AAL devices on the person's unicity and needs}

The design and implementation of AAL facilities must shift from a machine-centered to a person-centered process $[1,21]$. The machine-centered approach to device development is appropriate to scientific exploration of what is possible to achieve in new technology, but a person-centered process is necessary in assuring that the technologies developed fit well with human existence. AAL applications devoted to support people must be able to infer individual perception, choices and actions, and should simplify user access to future, remote and nearby social services overcoming impairments and language and cultural barriers. In this perspective, the user does not have to adapt or learn to use the device. Ideally, the device itself, while gathering information and meanings in the course of everyday activity, should build knowledge and practical ability to render the interaction interpretable and tailored to the users' abilities and disabilities [1]. This would result in a personal, friendly and familiar environment for the users, avoiding the threatening effects of unusual gadgets. The importance of considering the aesthetic side of this interaction has been also debated, as recent research pointed out the importance of shaping attractive, rich and acceptable relations between humans and AAL devices [10], thus improving well-being [37]. This would also prompt the need for "personalityzation", i.e. the need to design AAL technologies accounting for theoretical psychological personality constructs, such as openness, conscientiousness, extraversion, agreeableness, and neuroticism [38], which define more stable user's behavioral attributes with respect to continuously changing user's consumption and interests' profiles [2]. The shift from a mass-produced to a customized approach in designing AAL technologies has been emphasized by Bedaf et al. [7]. These authors showed that both elderly participants and caregivers expected these devices to be fully customizable on the needs and preferences of the user, being able to recognize her/his favorite objects in order to preserve the individual's (healthy) patterns of behavior, and expressed the desire that the device could be trained by the user to obtain a perfect match (see also [1]). Moreover, elderly participants and healthcare professionals shared the wish of AAL devices capable of engaging in meaningful conversations, e.g. with the device being able to comprehend and reply in a proper manner during interactions [7]. The need for devices able to perform sophisticated tasks, mimicking the care offered by human carers seems crucial for maximizing acceptance by seniors, who might be even keener to accept the devices than their caregivers [8].

\subsection{Complying with ethical and legal issues}

As outlined above, introducing AAL in the individual private space must comply with the fundamental ethical principles of autonomy, beneficence, non-maleficence, fidelity, justice, utility, and independence $[14,30]$ and must be preceded by an accurate assessment of the motivations, perspectives, preferences and concerns of the end users. These principles and 
insights in AAL are in line with the recent guidelines provided by the European Commission on Artificial Intelligence that specify the features that algorithms have to possess in order to be considered as ethical and trustworthy [60]. These requirements encompass: 1) Human Agency, i.e. the possibility to intervene easily on any decision the algorithm takes; 2) Robustness, i.e. the reliability and accuracy of the application; 3) Privacy, i.e. the need to guarantee a safe storage of private data; 4) Transparency, i.e. the possibility to track and monitor at any time decisions and algorithms exploited by the systems; 5) Non-discrimination, i.e. accessibility to everyone regardless to race, gender or age; 6) Well-being, i.e. sustainability of the systems in the environment and the society; 7) Accountability, i.e. the possibility to trace and promptly account for negative impacts of the systems.

The legal issues arising from the interaction of an individual with AAL devices represent a further theme of interest. Important legal aspects $[28,34]$ to be investigated are represented by, but not limited to 1) the need/duty to provide insurances for certain types of devices in case of accidents due to software errors or to the behavior of the device, 2) the definition of the extent to which these devices can replace humans in fulfilling certain activities and the range of tasks in which they can replace humans (labor law) and 3) the legal procedures to be followed in case of data leak or improper usage (data privacy law).These aspects should be evaluated in strict compliance with the standards associated with development, production, storage and distribution of medical devices as regulated by International Organization for Standardization [29].

\subsection{Reducing the risk of biases preventing validity of the intervention}

A further major issue is represented by ethics-related biases. Indeed, as in any other field of research, some systematic distortions in methods and data related to AAL technologies could compromise representativeness of the intervention. As described recently by Olteanu et al. [44], several stages (e.g. data collection, processing, analysis and evaluation) of the design and implementation of AAL technologies can lead to general biases, possibly affecting the construct, internal and external validity of the intervention itself. An instance of such biases is the population bias, i.e. a systematic distortion in demographics in a population of AAL users, which could affect external validity of findings gathered by using AAL technologies. Another example is represented by the normative bias, i.e. a bias that results from norms and expectations of acceptable patterns of behavior that could affect the user's behavior and evaluation of the proposed AAL technology. These examples, could even affect the fulfillment of the ethical principles described above, and pose challenges in the development of ethical AAL devices. Therefore, these biases need to be carefully addressed since the stage of research conception. Although it is unrealistic to cancel AAL-related biases, the development of standards and methodological best practices [44] is needed for advancing the ethical implementation of AAL devices. The first step in eliminating bias is recognizing that it may exist.

\subsection{Valorizing the psychological features of human-device interaction and multidisciplinary}

Finally, an important area of research is represented by the scientific investigation of the psychological aspects mediating the relationships between users and devices with a main focus on the ethical aspects of this interaction $[22,23]$. Indeed, during such a caring interaction the characteristics of the helper and of the recipient, and the complex exchanges occurring between them, can affect the readiness to seek help and reactions from receiving it [41]. In a 
dimension characterized by a fine balancing between dependence and autonomy, some personality and demographic features - such as dependent traits, self-esteem, attachment type, personal orientation in achieving contexts, age and gender - should be considered [2, 39-41]. To this purpose the use of semi-structured interviews, focus groups, questionnaires and experimental methods, involving users, care personnel and AAL developers, is strongly recommended to gain further knowledge on the ethical aspects associated to AAL applications. This would allow to emphasize the positive consequences of receiving help, such as belongingness and gratitude, and reduce the risk of the negative counterparts, such as dependency as a source of self-threat [41].

A multidisciplinary approach is also highly encouraged, based on highly qualified expert teams including psychologists, neurologists, geriatricians, neuroscientists, professional health coachers, health specialists, social workers, engineers and computer scientists, senior associations, hospitals, AAL companies and citizen scientists to work together and be involved at all stages of the device development [1]. This multidisciplinary approach together with the person-centered approach and the fine-tuning between dependence and autonomy in receiving and providing help, are important elements that can ensure the integration by design of ethical concepts into the implementation of AAL technologies. Ethical recommendations must be applied, as a process of standardization of AAL applications must include ethical recommendations as a standardization process to strengthen bridges between theory and practice.

We believe this perspective will prompt new research paradigm, and platforms, laying the foundation for future generations of personalized virtual coaches and robots to assist elderly people living independently at their home in accordance with the essential ethical principles.

Acknowledgments The research leading to these results has received funding from the EU 2020 research and innovation programme under grant agreement N. 769872 (EMPATHIC) and N. 823907 (MENHIR), the project SIROBOTICS that received funding from Ministero dell'Istruzione, dell'Università, e della Ricerca (MIUR), PNR 2015-2020, D.D. 1735, 13/07/2017, and the project ANDROIDS funded by the program V:ALERE2019 Università della Campania “Luigi Vanvitelli”, D.R. 906, 4/10/2019, prot. n. 157264, 17/10/2019.

Author contributions All authors contributed to the study conception and preparation. The first draft of the manuscript was written by Francesco Panico; Gennaro Cordasco, Carl Vogel, Luigi Trojano and Anna Esposito commented and revised the previous versions of the manuscript. All authors read and approved the final manuscript.

Funding Information Open access funding provided by Università degli Studi della Campania Luigi Vanvitelli within the CRUI-CARE Agreement.

\section{Compliance with ethical standards}

Conflict of interest The Authors declare that they have no conflict of interest.

Open Access This article is licensed under a Creative Commons Attribution 4.0 International License, which permits use, sharing, adaptation, distribution and reproduction in any medium or format, as long as you give appropriate credit to the original author(s) and the source, provide a link to the Creative Commons licence, and indicate if changes were made. The images or other third party material in this article are included in the article's Creative Commons licence, unless indicated otherwise in a credit line to the material. If material is not included in the article's Creative Commons licence and your intended use is not permitted by statutory regulation or exceeds the permitted use, you will need to obtain permission directly from the copyright holder. To view a copy of this licence, visit http://creativecommons.org/licenses/by/4.0/. 


\section{References}

1. 1st International Conference on Human Aspects of IT for the Aged Population, ITAP 2015 Held as Part of 17th International Conference on Human-Computer Interaction, HCI International 2015 (2015) Lecture Notes in Computer Science (including subseries Lecture Notes in Artificial intelligence and Lecture Notes in Bioinformatics), 9193, pp. 1-539.

2. Arazy O, Nov O, Kumar N (2015) Personalityzation: UI personalization, theoretical grounding in HCI and design research. ACM T Comput-Hum Int 7(2):43-69

3. Arking R (1998) The biology of aging, 2nd edn. Sinauer Associates, Sunderland

4. Baltes PB (1987) Theoretical propositions of life-span developmental psychology: on the dynamics between growth and decline. Dev Psychol 23(5):611-626

5. Baltes PB, Baltes MM (1990) Psychological perspectives on successful aging: the model of selective optimization with compensation.In P. Baltes \& M. Baltes (Eds.), Successful Aging: Perspectives from the Behavioral Sciences (European Network on Longitudinal Studies on Individual Development, pp. 1-34). Cambridge: CUP (1):1-34

6. Beauchamp TL, Childress JF (2001) Principles of biomedical ethics. Oxford University Press, USA

7. Bedaf S, Marti P, De Witte L (2017) What are the preferred characteristics of a service robot for the elderly? A multi-country focus group study with older adults and caregivers. Disabil Rehabil Assist Technol 31(3): $147-157$

8. Bedaf S, Marti P, Amirabdollahian F, de Witte L (2018) A multi-perspective evaluation of a service robot for seniors: the voice of different stakeholders. Disabil Rehabil Assist Technol 13(6):592-599

9. Bennett B, McDonald F, Beattie E, Carney T, Freckelton I, White B, Willmott L (2017) Assistive technologies for people with dementia: ethical considerations. Bull World Health Organ 95(11):749-755

10. Bertelsen O, Petersen MG, Pold S (2004) Aesthetic approaches to human-computer interaction. DAIMI Report Series, 33(572)

11. Blasco SA, Llobet DN, Koumanakos G (2019) Obtrusiveness considerations of AAL environments. In RADIO-Robots in Assisted Living (pp. 19-32). Springer, Cham

12. Bruder I, Heuer A, Karopka T, Schuldt J, Kosche K (2015) Experiences in developing and testing an ambient assisted living course for further education. In International Conference on Health Information Science (pp. 154-164). Springer, Cham

13. Carver J, Ganus A, Ivey JM, Plummer T, Eubank A (2016) The impact of mobility assistive technology devices on participation for individuals with disabilities. Disabil Rehabil Assist Technol 11(6):468-477

14. Cook AM (2009) Ethical issues related to the use/non-use of assistive technologies. Dev Disabil Bull 37 : $127-152$

15. Daniel KM, Cason CL, Ferrell S (2009) Emerging technologies to enhance the safety of older people in their homes. Geriatr Nurs 30(6):384-389

16. Demiris G, Hensel BK (2008) Technologies for an aging society: a systematic review of "smart home" applications. Yearb Med Inform 17(01):33-40

17. De-Rosende-Celeiro I, Torres G, Seoane-Bouzas M, Ávila A (2019) Exploring the use of assistive products to promote functional independence in self-care activities in the bathroom. PLoS One 14(4):ee0215002

18. D'Errico F, Leone G, Poggi I (2010) Types of help in the teacher's multimodal behavior. In International Workshop on Human Behavior Understanding. Springer, Berlin, Heidelberg, pp 125-139

19. Disabled Living Foundation (2019) Ethical issues with assistive technologies. https:// www.livingmadeeasy.org.uk/scenario.php?csid=43. Accessed 28 Nov 2019

20. Dziechciaz M, Filip R (2014) Biological psychological and social determinants of old age: bio-psychosocial aspects of human aging. Ann Agric Environ Med, 21(4).

21. Esposito A, Fortunati L, Lugano G (2014) Modeling emotion, behavior and context in socially believable robots and ICT interfaces. Cogn Comput 6(4):623-627

22. Esposito A et al (2019) Seniors' Acceptance of Virtual Humanoid Agents. In: Leone A, Caroppo A, Rescio G, Diraco G, Siciliano P (eds) Ambient Assisted Living. ForItAAL 2018. Lecture notes ine electrical engineering, vol 544. Springer, Cham

23. Esposito A, Amorese T, Cuciniello M, Riviello MT, Esposito AM, Troncone A, ... Cordasco G (2019) Elder user's attitude toward assistive virtual agents: the role of voice and gender. J Ambient Intell Humaniz Comput 1:8

24. Fiocco AJ, Yaffe K (2010) Defining successful aging: the importance of including cognitive function over time. Arch Neurol 67(7):876-880

25. Garçon L, Khasnabis C, Walker L, Nakatani Y, Lapitan J, Borg J, ... Velazquez Berumen A (2016) Medical and assistive health technology: meeting the needs of aging populations. Gerontologist 56(2):293-302

26. Gilbert SF (2000) Developmental biology, 6th edn. Sinauer Associates, Sunderland 
27. Hammel J (2003) Technology and the environment: supportive resource or barrier for people with developmental disabilities? Nurs Clin North Am 38(2):331-349

28. Huttunen A, Kulovesi J, Brace W, Lechner LG, Silvennoinen K, Kantola V (2010). Liberating intelligent machines with financial instruments. NJCL, 2

29. International Organization for Standardization 13485:2016. Medical devices — Quality Management systems - Requirements for regulatory purposes. https:/www.iso.org/standard/59752.html Accessed 21 Apr 2020

30. Kitchener KS (2000) Foundations of ethical practice, research, and teaching in psychology. Lawrence Erlbaum Associates, Inc., Mahwah

31. Kricos PB (2007) Hearing assistive technology considerations for older individuals with dual sensory loss. Trends Amplif 11(4):273-279

32. Lattanzio F, Mussi C, Scafato E, Ruggiero C, Dell'Aquila G, Pedone C, ... Carbonin PU (2010) Health care for older people in Italy: the ULISSE project (un link Informatico sui Servizi Sanitari Esistenti per l'anziano - a computerized network on health care services for older people). J Nutr Health Aging 14(3): $238-242$

33. Leone $\mathrm{G}$ (2012) Observing social signals in scaffolding interactions: how to detect when a helping intention risks falling short. Cogn Process 13(2):477-485

34. Leroux C, Labruto R, Boscarato C, Caroleo F, Günther J, Löffler S, Hilgendorf E Suggestion for a green paper on legal issues in robotics (2012). https://www.unipv-lawtech.eu/lang1/files/EUGreenPaper_ELS_IssuesInRobotics.pdf. Accessed 28 Nov 2019

35. Macklin R (2003) Bioethics, Vulnerability, and Protection. Bioethics 17(5-6):472-486

36. Martínez-Alcalá CI, Pliego-Pastrana P, Rosales-Lagarde A, Lopez-Noguerola JS, Molina-Trinidad EM (2016) Information and communication technologies in the care of the elderly: systematic review of applications aimed at patients with dementia and caregivers. JMIR Rehabil Assist Technol 3(1):e6

37. Martínez-Martí ML, Avia MD, Hernández-Lloreda MJ (2018) Effects of an appreciation of beauty randomized-controlled trial web-based intervention on appreciation of beauty and well-being. Psychol Aesthet Creat Arts 12(3):272-283

38. McCrae RR, Costa PT (1987) Validation of the five-factor model of personality across instruments and observers. J Pers Soc Psychol 52(1):81-90

39. Nadler A (1998) Relationship, esteem, and achievement perspectives on autonomous and dependent help seeking. In: Karabenick SA (ed) Strategic help seeking: implications for learning and teaching. Lawrence Erlbaum Associates Publishers, Mahwah, pp 61-93

40. Nadler A (2012) From help-giving to helping relations: belongingness and independence in social interaction. In: Deaux K, Snyder M (eds) The oxford handbook of personality and social psychology. Oxford University Press, New York, pp 394-418

41. Nadler A (2015) The other side of helping: seeking and receiving help. The Oxford handbook of prosocial behavior, 307-328

42. National Institute of Child Health and Human Development, Office of Communications (2018) What are some types of assistive devices and how are they used? https://www.nichd.nih.gov/health/topics/rehabtech/ conditioninfo/device. Accessed 28 Nov 2019

43. Novitzky P (2016) Ethics of ambient assisted living technologies for persons with dementia (Doctoral dissertation, Dublin City University)

44. Olteanu A, Castillo C, Diaz F, Kiciman E (2019) Social data: biases, methodological pitfalls, and ethical boundaries. Front Big Data 2:13

45. op den Akker H, op den Akker R, Beinema T, Banos O, Heylen D, Bedsted B, ... Hermens H (2018) Council of Coaches - a novel holistic behavior change coaching approach. Proceedings of the 4th International Conference on Information and Communication Technologies for Ageing Well and eHealth - volume 1: ICT4AWE (pages 219-226), Funchal Madeira, Portugal

46. Pedersen H, Söderström S, Kermit PS (2019) "The fact that I can be in front of others, I am used to being a bit behind": how assistive activity technology affects participation in everyday life. Disabil Rehabil Assist Technol 1:9

47. Poggi I, D'Errico F (2009) Social signals and the action-cognition loop. The case of overhelp and evaluation. In 2009 3rd International Conference on Affective Computing and Intelligent Interaction and Workshops (pp. 1-8). IEEE.

48. Rönnlund M, Nyberg L, Bäckman L, Nilsson LG (2005) Stability, growth, and decline in adult life span development of declarative memory: cross-sectional and longitudinal data from a population-based study. Psychol Aging 20(1):3-18

49. Salatino C, Pigini L, Van Kol MME, Gower V, Andrich R, Munaro G, ... Farina E (2017) A robotic solution for assisting people with MCI at home: preliminary tests of the ENRICHME system. Stud Health Technol Inform 242:484-491 
50. Scherer MJ, Federici S (2015) Why people use and don't use technologies: introduction to the special issue on assistive technologies for cognition/cognitive support technologies. NeuroRehabilitation 37(3):315-319

51. Schülke AM, Plischke H, Kohls NB (2010) Ambient assistive technologies (AAT): socio-technology as a powerful tool for facing the inevitable sociodemographic challenges? Philos Ethics Humanit Med 5(1):8

52. Seelbach C (1995) A perspective on early commercial applications of voice-processing technology for telecommunications and aids for the handicapped. P Natl Acad Sci U S A 92(22):9989-9990

53. Small N, Brooks H, Grundy A, Pedley R, Gibbons C, Lovell K, Bee P (2017) Understanding experiences of and preferences for service user and carer involvement in physical health care discussions within mental health care planning. BMC Psychiatry 17(1):138

54. The World Bank (2019) Life expectancy at birth. https://data.worldbank.org/indiecator/SP.DYN.LE00.IN. Accessed 28 Nov 2019

55. Torres MI, Olaso JM, Montenegro C, Santana R, Vázquez A, Justo R, ... Irvine M (2019) The EMPATHIC project: mid-term achievements. In Proceedings of the 12th ACM International Conference on Pervasive Technologies Related to Assistive Environments, 629-638

56. Valstar M, Baur T, Cafaro A, Ghitulescu A, Potard B, Wagner J, ... Pelachaud C (2016) Ask Alice: an artificial retrieval of information agent. In Proceedings of the 18th ACM International Conference on Multimodal Interaction (pp. 419-420)

57. Vandemeulebroucke T, de Casterle BD, Gastmans C (2018) How do older adults experience and perceive socially assistive robots in aged care: a systematic review of qualitative evidence. Aging Ment Health 22(2): $149-167$

58. Vandemeulebroucke T, de Casterle BD, Gastmans C (2018) The use of care robots in aged care: a systematic review of argument-based ethics literature. Arch Gerontol Geriatr 74:15-25

59. Vandemeulebroucke T, Dierckx de Casterlé B, Welbergen L, Massart M, Gastmans C (2019) The ethics of socially assistive robots in aged care. A focus group study with older adults in Flanders, Belgium J Gerontol $B$

60. High-Level Expert Group on Artificial Intelligence (2019). Ethics guidelines for trustworthy AI. https:// ec.europa.eu/digital-single-market/en/news/ethics-guidelines-trustworthy-ai. Accessed 28 Nov 2019

Publisher's note Springer Nature remains neutral with regard to jurisdictional claims in published maps and institutional affiliations. 